Sabrina Dalbosco Gadenz ${ }^{1}$ Luís Antônio Benvegnú ${ }^{2}$

\footnotetext{
${ }^{1}$ Programa de Residência Multiprofissional em Saúde da Família, Universidade Regional do Noroeste do Estado do Rio Grande do Sul. R. Boa Vista 401, Centro. 98.900-000 Santa Rosa RS Brasil. sabrinadalbosco@yahoo.com.br ${ }^{2}$ Programa de Residência em Medicina de Família e Comunidade, Departamento Ciências da Vida, Universidade Regional do Noroeste do Estado do Rio Grande do Sul.
}

\title{
Hábitos alimentares na prevenção de doenças cardiovasculares e fatores associados em idosos hipertensos
}

\author{
Eating habits in the prevention of cardiovascular diseases \\ and associated factors in elderly hypertensive individuals
}

Abstract Cardiovascular diseases are the leading cause of death and disability in the elderly. In this study, healthy eating habits and associated factors in the prevention of cardiovascular disease in elderly hypertensive individuals were identified. It involved a cross-sectional study gathering primary data in a family health unit in a city in the interior of the state of Rio Grande do Sul and 212 elderly hypertensive individuals participated in the study. A higher prevalence among the lower age bracket of elderly individuals, female, with little schooling and low income was revealed. Healthy eating habits among the elderly hypertensive individuals assessed are below those recommended in the prevention of cardiovascular disease. Social and economic factors, health characteristics and the use of health services influenced the adoption of healthy eating habits. The results of this study suggest that elderly hypertensive individuals find it difficult to adopt healthy eating habits. This contributes further to the definition of strategies for routine prevention and control of cardiovascular diseases in the elderly.

Key words Elderly, Eating habits Cardiovascular disease, Primary health care
Resumo As doenças cardiovasculares são a principal causa de morte e incapacidade em idosos. Nesta pesquisa, foram identificados hábitos alimentares saudáveis e fatores associados à prevenção de doenças cardiovasculares entre idosos hipertensos. Realizou-se um estudo transversal com coleta de dados primários em uma unidade de saúde da família pertencente a um município do interior do Rio Grande do Sul. Participaram do estudo 212 idosos hipertensos. Verificou-se maior prevalência de idosos jovens, do sexo feminino, com poucos anos de estudo e de baixa renda. Os hábitos alimentares saudáveis dos idosos hipertensos avaliados estão aquém daqueles preconizados. Fatores socioeconômicos, características de saúde e utilização dos serviços de saúde influenciaram na prática de hábitos alimentares saudáveis. Os resultados desse estudo sugerem que os idosos hipertensos estão encontrando dificuldade para adotar hábitos alimentares saudáveis; contribuem ainda para a definição de estratégias para o trabalho cotidiano de prevenção e controle das doenças cardiovasculares em idosos.

Palavras-chave Idoso, Hábitos alimentares, Doenças cardiovasculares, Atenção primária à saúde 


\section{Introdução}

As doenças cardiovasculares (DCV) permanecem como principal causa de morte e incapacidade em idosos, mesmo após diminuição do hábito de tabagismo e maior acesso desta população à atenção primária ${ }^{1,2}$. Além disso, o Brasil tem uma população idosa em rápido crescimento, aumentando de $8,6 \%$ para $11 \%$ em dez anos ${ }^{3}$. A hipertensão arterial sistêmica (HAS) é o principal fator de risco para o desenvolvimento de DCV em idosos ${ }^{4}$.

A alimentação saudável tem importante impacto na prevenção e tratamento das DCV, assim como pequenas melhorias também trazem relevantes benefícios na diminuição do risco cardiovascular ${ }^{1,5-7}$. Em muitos países tem sido relatadas alterações nos padrões de hábitos alimentares $^{8}$. No Brasil, tem-se observado que a dieta vigente apresenta maior participação de alimentos com excesso de calorias provenientes de açúcares livres e de gorduras saturadas e menor presença de frutas, legumes e verduras ${ }^{9}$. Portanto, é de extrema importância identificar os hábitos alimentares dos idosos, a fim de promover melhores estratégias de prevenção de DCV.

As políticas púbicas de saúde no Brasil têm focalizado estratégias para combater as doenças crônicas não transmissíveis (DCNT) por meio de ações intersetoriais de caráter preventivo e de promoção à saúde. Entre estas ações estão o monitoramento de fatores de risco e a atenção à saúde centrada em dietas saudáveis, prática de atividade física, redução do tabagismo e do etilismo ${ }^{2}$. O Ministério da Saúde recentemente publicou o plano de ações estratégicas para o enfrentamento das DCNT, visando preparar o país para enfrentar e deter, nos próximos dez anos, essas doenças e as morbidades a elas relacionadas ${ }^{10}$.

A Organização Mundial da Saúde (OMS) e a American Heart Association (AHA) definiram estratégias para redução do risco de doenças cardiovasculares. Entre elas, está a adoção de uma alimentação rica em frutas e vegetais, dando preferência aos grãos integrais, alimentos ricos em fibras, peixes, carnes magras e produtos lácteos com baixo teor de gordura. Também, minimizar a ingestão de açúcar e preferir alimentos com pouco $\mathrm{sal}^{7,11}$.

O objetivo deste estudo foi identificar hábitos alimentares saudáveis para prevenção de DCV em idosos hipertensos e avaliar fatores associados.

\section{Métodos}

Trata-se de um estudo epidemiológico transversal com coleta de dados primários entre outubro e dezembro de 2011 em uma unidade de saúde da família pertencente a um município do interior do Rio Grande do Sul.

A população do estudo foi constituída pelos idosos (60 anos ou mais) hipertensos residentes em uma área com cobertura integral de agentes comunitários de saúde (ACS). A identificação deuse pelo levantamento de todos os idosos com diagnóstico de hipertensão, registrados no Sistema de Informação da Atenção Básica (SIAB) e/ou que possuíam cadastro de medicamento na unidade de saúde, totalizando 270 indivíduos. Para o estudo dessa população, trabalhou-se com uma amostra representativa dos idosos hipertensos.

O cálculo do tamanho amostral foi realizado para avaliar, com um nível de confiança de 95\%, hábitos alimentares com frequência de $12 \%{ }^{12}$. A margem de erro considerada foi de 2 pontos percentuais. Para suprir possíveis perdas ou recusas foi acrescentado $10 \%$, resultando numa amostra necessária de 234 indivíduos. A seleção da amostra foi realizada por meio de amostragem aleatória simples com o auxílio do Programa Epi Info 6.04 (Centers for Disease Control and Prevention, Atlanta, Estados Unidos). Foram registradas 5 perdas por recusa, 4 por óbito, 8 por mudança de endereço, 1 por hospitalização e outro havia sido institucionalizado. Ainda houve 3 idosos acamados que foram excluídos. Portanto, foram entrevistados efetivamente 212 idosos, conferindo uma perda de $9,4 \%$ da amostra inicial.

A coleta de dados foi realizada no domicílio dos idosos por entrevistadores previamente treinados. Nas visitas foi aplicado um questionário pré-codificado e pré-testado em estudo piloto com 20 idosos residentes em outra área do município. Nos domicílios também foram aferidas as medidas antropométricas (peso e estatura) para posteriormente calcular o Índice de Massa Corporal (IMC).

A verificação do peso corporal foi feita em balança digital portátil G-TECH com variação de $0,1 \mathrm{Kg}$ e capacidade até $180 \mathrm{Kg}$ devidamente aferida e colocada em superfície nivelada, a pessoa estava com roupas leves e descalça. A estatura foi verificada por meio de fita inelástica com variação em milímetros fixada em parede lisa e sem rodapés e com o auxílio de um esquadro de madeira. A pessoa estava descalça, com a coluna ereta e encostada na parede e os calcanhares um ao lado do outro e juntos à parede ${ }^{13}$. A classificação 
do estado nutricional pelo IMC foi feita de acordo com a recomendação de Lipschitz ${ }^{14}$ : baixo peso $\left(\mathrm{IMC}<22 \mathrm{~kg} / \mathrm{m}^{2}\right.$ ), eutrofia (IMC entre 22 e $27 \mathrm{~kg} /$ $\mathrm{m}^{2}$ ) e excesso de peso $\left(\mathrm{IMC}>27 \mathrm{~kg} / \mathrm{m}^{2}\right)$.

O desfecho analisado foi de hábitos alimentares saudáveis para prevenção de doenças cardiovasculares. Considerou-se hábito alimentar saudável quem consumia frutas e hortaliças diariamente. Estes foram avaliados por meio de um questionário de frequência alimentar (QFA) (nunca ou menos de uma vez por mês, menos que uma vez por semana, uma vez por semana, duas a três vezes por semana, quatro a seis vezes por semana ou diariamente). Também foi considerado hábito alimentar saudável para prevenção de DCV quem consumia pão integral (qual o tipo de pão que o senhor consome com maior frequência?), leite com pouca/sem gordura (Se o senhor bebe leite, qual o tipo que normalmente consome?), preparava as carnes de forma não frita (De que forma, geralmente, as carnes são preparadas?) e usava gordura vegetal para cozinhar (Qual é o tipo de gordura normalmente usada para cozinhar na sua casa?). Também foi avaliado por meio do QFA o consumo de peixe (nunca ou menos de uma vez por mês, menos que uma vez por semana, uma vez por semana, duas a três vezes por semana, quatro a seis vezes por semana ou diariamente). Para considerar hábitos alimentares saudáveis na prevenção de DCV foram seguidas as recomendações da Organização Mundial da Saúde $(\mathrm{OMS})^{11}$ e da American Heart Association (AHA) ${ }^{7}$.

As variáveis de exposição estudadas foram as socioeconômicas e as demográficas (faixa etária, sexo, aposentadoria, situação conjugal, escolaridade em anos de estudo e renda familiar em salários mínimos), as características de saúde (prática de atividade física, tabagismo, estado nutricional, diabete mellitus, percepção do apetite, percepção da saúde bucal, perda de peso involuntária), utilização de serviços de saúde (plano de saúde, realização de consulta médica no último ano, número de consultas médicas no último ano, se já realizou consulta com nutricionista, local da consulta com nutricionista, forma de agendamento da consulta com nutricionista) e práticas alimentares Quem normalmente prepara a comida para o senhor (a)? Alguém faz as refeições junto com o senhor(a)? Quem faz a compra dos alimentos na sua casa? Onde o senhor normalmente faz as refeições? Qual o principal motivo para escolher os alimentos que vai consumir? Número diário de refeições (foi considerada refeição quem informou ter ingerido algum alimento em uma das refeições principais, isto é, desjejum, lanche da manhã, almoço, lanche da tarde, jantar e ceia, pequenos "beliscos" não foram considerados como refeição), O senhor(a) usa tempero pronto, industrializado? O senhor(a) consome embutidos? O senhor (a) adiciona sal às refeições prontas? Com que frequência o senhor (a) consome doces? (nunca ou menos de uma vez por mês, menos que uma vez por semana, uma vez por semana, duas a três vezes por semana, quatro a seis vezes por semana ou diariamente. Para a análise, considerou-se quem consumia doces diariamente e não diariamente). Com que frequência o senhor (a) consome refrigerantes? (nunca ou menos de uma vez por mês, menos que uma vez por semana, uma vez por semana, duas a três vezes por semana, quatro a seis vezes por semana ou diariamente. Para a análise, considerou-se quem bebia refrigerante uma vez na semana ou menos ou mais do que uma vez na semana)].

A condição de fumante foi atribuída a todos que relataram fumar, independentemente da frequência e intensidade do hábito. Foi considerado diabético quem referiu a doença, uso de hipoglicemiante oral ou insulina. Foi considerado ser praticante de atividade física quem praticava todos os dias da semana no mínimo trinta minutos conforme recomendado pela OMS para prevenção de doenças cardiovasculares ${ }^{11}$. Para avaliar a percepção do apetite e da saúde bucal foi abordado em forma de escala (ótimo/bom/regular/mau/péssimo).

A estruturação do banco de dados foi feita no programa Epi Info 3.5.3 com dupla entrada para correção de possíveis erros.

A análise estatística consistiu em uma análise descritiva dos dados através do estudo das medidas de tendência central e dispersão com obtenção das frequências absolutas e relativas para as categorias. Para análise bivariada foi utilizado o teste Qui-quadrado de Pearson e o teste de Fisher. Adotou-se como nível de significância estatística o valor de $\mathrm{p}<0,05$ para todas as comparações.

Na pesquisa foram observados todos os preceitos éticos da Resolução 196/1996 ${ }^{15}$ do Conselho Nacional de Saúde e o projeto foi aprovado pelo Comitê de Ética em Pesquisa da Unujuí .

\section{Resultados}

Do total dos idosos entrevistados, $123(58,0 \%)$ eram do sexo feminino. A maioria (59,9\%) estava na faixa-etária dos 60-69 anos e eram casados $(62,3 \%)$. A renda familiar predominante entre os idosos foi igual ou menor do que dois salários 
mínimos, contando que 80,2\% estavam aposentados. Com relação à escolaridade, em torno de $80,0 \%$ dos idosos estudaram cinco anos ou menos. A Tabela 1 descreve as características socioeconômicas e demográficas estratificadas pelo sexo.

Em relação às características de saúde, 192 $(90,6 \%)$ dos idosos não praticavam atividade física conforme recomendado e 137 (64,6\%) estavam com excesso de peso. A prevalência de tabagismo foi de 14,2\%. Além de hipertensos, 54 $(25,5 \%)$ idosos também eram diabéticos. A maioria relatou que a saúde bucal $(53,8 \%)$ e o apetite $(64,6 \%)$ estavam bons ou ótimos, e 43 (20,7\%) relataram perda de peso involuntária (Tabela 2). Os idosos com 80 anos ou mais foram aqueles que relataram maior perda de peso involuntária $(34,6 \%)$, e também os que menos disseram que o apetite $(53,8 \%)$ e a saúde bucal $(50,0 \%)$ estavam bons ou ótimos (dados não apresentados em tabela).

A grande maioria dos idosos (86,8\%) relatou que fizeram consulta médica no último ano, e dentre estes, 155 (84,2\%) se consultaram duas vezes ou mais. Da totalidade dos idosos, 47 $(22,2 \%)$ possuíam plano de saúde. Mais do que a metade dos entrevistados $(59,0 \%)$ nunca consultaram com nutricionista. Entre aqueles que consultaram $78(89,7 \%)$ relataram que foi na unidade de saúde da família e 74 (85,1\%) disseram que foram encaminhados à nutricionista por outros profissionais de saúde (Tabela 3 ).

Sobre a compra e o preparo dos alimentos, $128(60,4 \%)$ e $114(53,8 \%)$, respectivamente, relataram que eles próprios o faziam. A maioria realizava as refeições em casa $(96,2 \%)$ e com companhia $(86,8 \%)$. O principal motivo citado para a escolha dos alimentos foi o hábito $(35,4 \%)$, seguido pelo preço (31,6\%). A prevalência dos idosos que faziam cinco refeições diárias ou mais foi $25,0 \%$. O uso de temperos industrializados foi relatado por $145(68,4 \%)$ dos entrevistados, o consumo de embutidos por 134 (63,2\%). Somente $11(5,2 \%)$ dos idosos relataram que adicionavam sal às refeições prontas. A prevalência dos idosos que consumiam refrigerantes mais do que uma vez na semana foi de 19,3\%. O consumo diário de doces ficou com prevalência próxima a 10\%. (Tabela 4). O consumo de peixe, no mínimo uma vez por semana, foi referido por $33(15,6 \%)$ dos entrevistados. Dentre estes, apenas $11(33,3 \%)$ con-

Tabela 1. Distribuição das características socioeconômicas e demográficas entre idosos hipertensos de acordo com o sexo. Município de Santa Rosa, Rio Grande do Sul, Brasil, 2011.

\begin{tabular}{|c|c|c|c|}
\hline Variáveis & $\begin{array}{c}\text { Feminino } \\
\mathbf{n}(\%)\end{array}$ & $\begin{array}{c}\text { Masculino } \\
\text { n (\%) }\end{array}$ & Valor de $\mathrm{p}$ \\
\hline Faixa etária (anos) & & & 0,205 \\
\hline $60-69$ & $69(56,1)$ & $58(65,1)$ & \\
\hline $70-79$ & $35(28,5)$ & $24(27,0)$ & \\
\hline$\geq 80$ & $19(15,4)$ & $7(7,9)$ & \\
\hline Escolaridade (anos) & & & 0,003 \\
\hline Sem escolaridade & $21(17,1)$ & $11(12,3)$ & \\
\hline $1-4$ & $64(52,0)$ & $33(37,1)$ & \\
\hline $5-10$ & $35(28,5)$ & $32(36,0)$ & \\
\hline$\geq 11$ & $3(2,4)$ & $13(14,6)$ & \\
\hline Aposentadoria & & & 0,008 \\
\hline $\operatorname{Sim}$ & $91(74,0)$ & $79(88,8)$ & \\
\hline Não & $32(26,0)$ & $10(11,2)$ & \\
\hline Situação Conjugal & & & $<0,001$ \\
\hline Casado & $52(42,3)$ & $80(89,9)$ & \\
\hline Separado & $9(7,3)$ & $1(1,1)$ & \\
\hline Solteiro & $6(4,9)$ & $2(2,2)$ & \\
\hline Viúvo & $56(45,5)$ & $6(6,8)$ & \\
\hline Renda Familiar (salários mínimos) ${ }^{*}$ & & & 0,005 \\
\hline$\leq 1$ & $40(32,8)$ & $14(15,8)$ & \\
\hline $1,1-2$ & $48(39,4)$ & $31(34,8)$ & \\
\hline $2,1-5$ & $27(22,1)$ & $36(40,4)$ & \\
\hline$>5$ & $7(5,7)$ & $8(9,0)$ & \\
\hline
\end{tabular}

"salário mínimo: R\$ 545,00 (um não soube responder) 
Tabela 2. Características de saúde entre idosos hipertensos de acordo com o sexo. Município de Santa Rosa, Rio Grande do Sul, Brasil 2011.

\begin{tabular}{|c|c|c|c|}
\hline Variáveis & $\begin{array}{c}\text { Feminino } \\
\mathbf{n}(\%)\end{array}$ & $\begin{array}{c}\text { Masculino } \\
\text { n (\%) }\end{array}$ & Valor de p \\
\hline Tabagismo (fumante atual) & & & 0,003 \\
\hline Sim & $10(8,1)$ & $20(22,5)$ & \\
\hline Não & $113(91,9)$ & $69(77,5)$ & \\
\hline Atividade Física & & & 0,445 \\
\hline Sim & $10(8,1)$ & $10(11,2)$ & \\
\hline Não & $113(91,9)$ & $79(88,8)$ & \\
\hline Estado Nutricional & & & 0,053 \\
\hline Baixo peso & $13(10,6)$ & $6(6,7)$ & \\
\hline Eutrófico & $25(20,3)$ & $31(34,8)$ & \\
\hline Excesso de peso & $85(69,1)$ & $52(58,4)$ & \\
\hline Diabete Mellitus & & & 0,070 \\
\hline $\operatorname{Sim}$ & $37(30,1)$ & $17(19,1)$ & \\
\hline Não & $86(69,9)$ & $72(80,9)$ & \\
\hline Apetite & & & 0,014 \\
\hline Bom/ótimo & $70(56,9)$ & $67(75,3)$ & \\
\hline Regular & $43(35,0)$ & $20(22,5)$ & \\
\hline Mal/ Péssimo & $10(8,1)$ & $2(2,2)$ & \\
\hline Saúde bucal & & & 0,378 \\
\hline Bom/ótimo & $71(57,7)$ & $43(48,3)$ & \\
\hline Regular & $41(33,4)$ & $35(39,3)$ & \\
\hline Mal/ Péssimo & $11(8,9)$ & $11(12,4)$ & \\
\hline Perda de peso involuntária* & & & 0,996 \\
\hline $\operatorname{Sim}$ & $25(20,7)$ & $18(20,7)$ & \\
\hline Não & $96(79,3)$ & $69(79,3)$ & \\
\hline
\end{tabular}

4 não souberam responder

Tabela 3. Uso de serviços de saúde por idosos hipertensos de acordo com o sexo. Município de Santa Rosa, Rio Grande do Sul, Brasil, 2011.

\begin{tabular}{|c|c|c|c|}
\hline Variáveis & $\begin{array}{c}\text { Feminino } \\
\mathbf{n}(\%)\end{array}$ & $\begin{array}{c}\text { Masculino } \\
\text { n }(\%)\end{array}$ & Valor de $\mathrm{p}$ \\
\hline Já consultou com nutricionista & & & 0,666 \\
\hline Sim & $52(42,3)$ & $35(39,3)$ & \\
\hline Não & $71(57,7)$ & $54(60,7)$ & \\
\hline Local da consulta com nutricionista ${ }^{*}$ & & & 0,088 \\
\hline Unidade de saúde & $49(94,3)$ & $29(83,3)$ & \\
\hline Convênio/ particular & $3(5,7)$ & $6(16,7)$ & \\
\hline Agendamento da consulta com nutricionista & & & 0,637 \\
\hline Referenciado & $45(86,5)$ & $29(82,9)$ & \\
\hline Procura espontânea & $7(13,5)$ & $6(17,1)$ & \\
\hline Realização de consulta médica no último ano & & & 0,031 \\
\hline $\operatorname{Sim}$ & $112(91,1)$ & $72(80,9)$ & \\
\hline Não & $11(8,9)$ & $17(19,1)$ & \\
\hline Número de consultas médicas ${ }^{* *}$ & & & 0,787 \\
\hline Uma & $17(15,2)$ & $12(16,7)$ & \\
\hline Duas ou mais & $95(84,8)$ & $60(83,3)$ & \\
\hline Plano de Saúde & & & 0,562 \\
\hline Sim & $29(23,6)$ & $18(20,2)$ & \\
\hline Não & $94(76,4)$ & $71(79,8)$ & \\
\hline
\end{tabular}

$n=87 ; " n=184$ 
Tabela 4. Práticas alimentares entre idosos hipertensos. Município de Santa Rosa, Rio Grande do Sul, Brasil, 2011.

\begin{tabular}{|c|c|c|c|}
\hline Variáveis & $\begin{array}{c}\text { Feminino } \\
\mathbf{n}(\%)\end{array}$ & $\begin{array}{c}\text { Masculino } \\
\text { n }(\%)\end{array}$ & Valor de $\mathrm{p}$ \\
\hline Quem prepara as refeições & & & $<0,001$ \\
\hline Idoso & $94(76,4)$ & $20(22,5)$ & \\
\hline Familiar/Cuidador/outros & $29(23,6)$ & $69(77,5)$ & \\
\hline Quem compra os alimentos & & & 0,028 \\
\hline Idoso & $82(66,7)$ & $46(51,7)$ & \\
\hline Familiar/Cuidador/outros & $41(33,3)$ & $43(48,3)$ & \\
\hline Local das refeições & & & 0,060 \\
\hline Em casa & $121(98,4)$ & $83(93,3)$ & \\
\hline Outros lugares & $2(1,6)$ & $6(6,7)$ & \\
\hline Motivo principal na escolha & & & 0,934 \\
\hline Hábito & $44(35,8)$ & $31(34,8)$ & \\
\hline Preço & $37(30,1)$ & $30(33,7)$ & \\
\hline Saudável & $27(22,0)$ & $17(19,1)$ & \\
\hline Outros & $15(12,1)$ & $11(12,4)$ & \\
\hline Companhia nas refeições & & & 0,018 \\
\hline Sim & $101(82,1)$ & $83(93,3)$ & \\
\hline Não & $22(17,9)$ & $6(6,7)$ & \\
\hline Número de refeições diárias & & & 0,045 \\
\hline 5 ou mais & $37(30,1)$ & $16(18,0)$ & \\
\hline Menos do que 5 & $86(69,9)$ & $73(82,0)$ & \\
\hline Uso de tempero industrializado & & & 0,736 \\
\hline Sim & $83(67,5)$ & $62(69,7)$ & \\
\hline Não & $40(32,5)$ & $27(30,3)$ & \\
\hline Consumo de embutidos & & & 0,280 \\
\hline Sim & $74(60,2)$ & $60(67,4)$ & \\
\hline Não & $49(39,8)$ & $29(32,6)$ & \\
\hline Adição de sal à refeição pronta & & & 0,523 \\
\hline Sempre ou às vezes & $6(4,9)$ & $5(5,6)$ & \\
\hline Nunca & $117(95,1)$ & $84(94,4)$ & \\
\hline Consumo de refrigerante & & & 0,041 \\
\hline Mais do que uma vez na semana & $18(14,6)$ & $23(25,8)$ & \\
\hline Uma vez na semana ou menos & $105(85,4)$ & $66(74,2)$ & \\
\hline Consumo de doces & & & 0,051 \\
\hline Diariamente & $8(6,5)$ & $13(14,6)$ & \\
\hline Não diariamente & $115(93,5)$ & $76(85,4)$ & \\
\hline
\end{tabular}

${ }^{*} \mathrm{n}=87 ;{ }^{* *} \mathrm{n}=184$

sumiam semanalmente duas vezes ou mais - frequência recomendada para prevenção de DCV (dados não apresentados em tabela).

Para todos os hábitos alimentares saudáveis para prevenção de DCV analisados não houve diferença significativa entre os idosos mais jovens ( $<80$ anos) e os longevos ( $\geq 80$ anos). Ter mais anos de estudos associou-se positivamente com maiores prevalências no consumo de frutas e hortaliças. Já a renda familiar foi associada de forma significativa apenas com o consumo diário de hortaliças. Na maioria dos hábitos alimentares saudáveis, os não fumantes apresentaram prevalências significativamente maiores do que os fumantes. Ser praticante de atividade física não foi associado de forma significativa com melhores hábitos alimentares. Os idosos com excesso de peso tiveram maior prevalência de hábitos alimentares saudáveis quando comparados aos que os que não tinham excesso de peso. Foi associado aos que referiram o apetite bom ou ótimo maior prevalência de consumo de frutas e hortaliças. Maiores prevalências de hábitos alimentares saudáveis também foram observadas entre os que já haviam consultado com nutricionista quando comparado aos que não. Quem comprava os alimentos e preparava as refeições não influenciou nos hábitos alimenta- 
res saudáveis. Os idosos que referiram o saudável como principal motivo para a escolha dos alimentos apresentaram maior frequência na maioria dos hábitos alimentares saudáveis analisados. Entre os sexos não foram evidenciadas diferenças significativas nos hábitos alimentares saudáveis na prevenção de DCV (Tabela 5).

\section{Discussão}

Os resultados observados no presente estudo apontam para aspectos relevantes ao sinalizar que os hábitos alimentares saudáveis dos idosos hipertensos avaliados estão aquém daqueles preconizados. Nossos achados também sugerem que fatores socioeconômicos, características de saúde e utilização dos serviços de saúde influenciam na prática de hábitos alimentares saudáveis para prevenção de DCV entre idosos hipertensos. Esse é um dos primeiros estudos no país que descreve hábitos alimentares de idosos hipertensos e identifica fatores associados.

O perfil socioeconômico e demográfico dos idosos estudados é semelhante ao encontrado em outros estudos realizados no país, que é característico da população idosa brasileira ${ }^{16-18}$. Verificou-se a prevalência de idosos jovens, predomínio do sexo feminino, com poucos anos de estudo e de baixa renda. As idosas apresentaram estas vulnerabilidades socioeconômicas mais acentuadas. Embora tenha sido estudada somente uma área de abrangência da ESF, os dados aqui apresentados podem retratar a realidade de uma grande parcela de idosos dos municípios brasileiros.

Os resultados desta pesquisa devem ser interpretados no contexto de algumas limitações. O QFA aplicado não avaliou o tamanho das porções de alimentos consumidos. Outro fator diz respeito ao método do QFA, que essencialmente depende da memória dos entrevistados, consti-tuindo uma limitação ainda maior em idosos. No entanto, a aplicação do questionário foi realizada por entrevistadores treinados, atenuando estas limitações. Também não foi avaliada a forma de preparo do consumo de peixe, o que limitou a classificação deste alimento como hábito alimentar saudável. Além disso, há de se considerar como limitação do estudo as inerentes ao delineamento transversal, como o viés de sobrevivência e a causalidade reversa.

O hábito e o preço foram as principais motivações referidas pelos idosos para a escolha dos alimentos. Em Minas Gerais, 22,4\% dos hipertensos orientados quanto à importância da in- gestão de uma alimentação saudável, afirmaram não seguir tais orientações devido aos hábitos e aos fatores econômicos ${ }^{19}$. Apesar da modificação de hábitos alimentares ser uma das principais estratégias para prevenção de DCV, parece que apenas orientações quanto a isso não tem feito com que os idosos incorporem hábitos alimentares mais saudáveis. Os fatores socioculturais e econômicos parecem prevalecer entre os idosos na hora da escolha dos alimentos. Além do mais, ficou evidente que os idosos que consideravam o ser saudável como principal motivação, tiveram maiores prevalências dos hábitos alimentares saudáveis avaliados.

Fazer refeições de maneira irregular tem sido associado ao aumento de fatores de risco cardiovascular em idosos ${ }^{20,21}$. O maior fracionamento das refeições é uma importante medida de prevenção e controle de DCV ${ }^{19,22}$. Contudo, o mínimo recomendado de cinco refeições por dia pelo Manual da Pessoa Idosa do Ministério da Saúde ${ }^{23}$ não era realizado pela maioria dos idosos entrevistados. Isso demanda para os profissionais de saúde um maior enfoque no fracionamento de refeições dos idosos. Observamos também que o fracionamento das refeições foi associado de forma significativa com maior frequência de consumo diário de frutas, o mesmo foi encontrado por Sierra-Johnson et al. ${ }^{20}$. Provavelmente, os idosos que fracionam melhor suas refeições, possuem também melhores hábitos alimentares.

O efeito da diminuição da ingestão de sódio no controle da hipertensão e na diminuição do risco de eventos cardiovasculares tende a ser ainda maior em idosos e hipertensos ${ }^{11}$. Em nosso estudo, a elevada prevalência de consumo de temperos industrializados e embutidos indicam um excesso na ingestão de sódio, mesmo que somente $5,2 \%$ referiram adicionar sal às refeições prontas. A implementação de políticas para redução do sódio nos alimentos é considerada uma das estratégias mais seguras e rentáveis na redução do risco de $\mathrm{DCV}^{24}$. Além disso, a elevada prevalência de consumo desses alimentos reforça a necessidade de orientações nutricionais direcionadas a essa população, uma vez que a falta de conhecimento e informação sobre a composição nutricional dos alimentos também pode determinar um excesso no consumo.

Da mesma forma, foi verificada uma alimentação rica em gordura saturada e colesterol. A maioria utilizava gordura animal para cozinhar, preparava as carnes de forma frita e consumia leite com alto teor de gordura. Nenhum fator socioeconômico foi associado a esses achados. 
Tabela 5. Hábitos alimentares saudáveis na prevenção de doenças cardiovasculares segundo variáveis socioeconômicas, características de saúde, uso de serviços de saúde e práticas alimentares. Município de Santa Rosa, Rio Grande do Sul, Brasil, 2011.

\begin{tabular}{|c|c|c|c|c|c|c|}
\hline Variáveis & Hortaliças & Frutas & $\begin{array}{c}\text { Pão } \\
\text { integral }\end{array}$ & $\begin{array}{c}\text { Gordura } \\
\text { Vegetal }\end{array}$ & $\begin{array}{l}\text { Leite pouco/ } \\
\text { sem gordura }\end{array}$ & $\begin{array}{c}\text { Carnes não } \\
\text { fritas }\end{array}$ \\
\hline Total n (\%) & $162(76,4)$ & $147(69,3)$ & $42(19,8)$ & $87(41,0)$ & $39(21,3)$ & $41(19,3)$ \\
\hline \multicolumn{7}{|l|}{ Sexo [valor de $\left.\mathrm{p}^{*}\right]$} \\
\hline Feminino & $92(74,8)$ & $91(74,0)$ & $29(23,6)$ & $50(40,7)$ & $22(19,8)$ & $26(21,1)$ \\
\hline Masculino & $70(78,7)$ & $56(62,9)$ & $13(14,6)$ & $37(41,6)$ & $17(23,6)$ & $15(16,9)$ \\
\hline \multicolumn{7}{|l|}{ Faixa etária [valor de p*] } \\
\hline$<80$ anos & $144(77,4)$ & $131(70,4)$ & $38(20,4)$ & $75(40,3)$ & $37(23,1)$ & $36(19,4)$ \\
\hline$\geq 80$ anos & $18(69,2)$ & $16(61,5)$ & $4(15,4)$ & $12(46,2)$ & $2(8,7)$ & $5(19,2)$ \\
\hline Escolaridade [valor de $\mathrm{p}^{*}$ ] & $\mathrm{p}<0,05$ & $\mathrm{p}<0,05$ & & & & \\
\hline $0-4$ anos & $86(66,7)$ & $80(62,0)$ & $25(19,4)$ & $48(37,2)$ & $22(20,4)$ & $25(19,4)$ \\
\hline$\geq 5$ anos & $76(91,6)$ & $67(80,7)$ & $17(20,5)$ & $39(47,0)$ & $17(22,7)$ & $16(19,3)$ \\
\hline Renda Familiar [valor de $\mathrm{p}^{*}$ ] & $\mathrm{p}<0,05$ & & & & & \\
\hline$\leq 2$ salários mínimo & $35(64,8)$ & $33(61,1)$ & $12(22,2)$ & $57(42,9)$ & $9(18,4)$ & $10(18,5)$ \\
\hline >2 salários mínimo & $26(92,9)$ & $24(85,7)$ & $4(14,3)$ & $30(38,5)$ & $5(25,0)$ & $7(25,0)$ \\
\hline Tabagismo [valor de $\mathrm{p}^{*}$ ] & $\mathrm{p}<0,05$ & $\mathrm{p}<0,05$ & $\mathrm{p}<0,05^{\star *}$ & & & $\mathrm{p}<0,05^{\star *}$ \\
\hline $\operatorname{Sim}$ & $17(56,7)$ & $13(43,3)$ & $1(3,3)$ & $9(30,0)$ & $4(14,8)$ & $2(6,7)$ \\
\hline Não & $145(79,7)$ & $134(73,6)$ & $41(22,5)$ & $78(42,9)$ & $35(22,4)$ & $39(21,4)$ \\
\hline \multicolumn{7}{|l|}{ Atividade Física [valor de $\mathrm{p}^{*}$ ] } \\
\hline Sim & $18(90,0)$ & $17(85,0)$ & $3(15,0)$ & $9(45,0)$ & $7(35,0)$ & $1(5,0)$ \\
\hline Não & $144(75,0)$ & $130(67,7)$ & $39(20,3)$ & $78(40,6)$ & $32(19,6)$ & $40(20,8)$ \\
\hline Estado Nutricional [valor de $\mathrm{p}^{*}$ ] & $\mathrm{p}<0,05$ & $\mathrm{p}<0,05$ & & & $\mathrm{p}<0,05$ & \\
\hline Excesso de peso & $111(81,0)$ & $103(75,2)$ & $32(23,4)$ & $61(44,5)$ & $31(25,6)$ & $29(21,2)$ \\
\hline Sem excesso de peso & $51(68,0)$ & $44(58,7)$ & $10(13,3)$ & $26(34,7)$ & $8(12,9)$ & $12(16,0)$ \\
\hline Diabete Melitus [valor de $\mathrm{p}^{*}$ ] & & & $\mathrm{p}<0,05$ & & $\mathrm{p}<0,05$ & \\
\hline Sim & $38(70,4)$ & $42(77,8)$ & $22(40,7)$ & $23(42,6)$ & $16(37,2)$ & $10(18,5)$ \\
\hline Não & $124(78,5)$ & $105(66,5)$ & $20(12,7)$ & $64(40,5)$ & $23(16,4)$ & $31(19,6)$ \\
\hline Apetite [valor de $\mathrm{p}^{*}$ ] & $\mathrm{p}<0,05$ & $\mathrm{p}<0,05$ & & & & \\
\hline Bom/ótimo & $112(81,8)$ & $105(76,6)$ & $31(22,6)$ & $58(42,3)$ & $21(17,9)$ & $29(21,2)$ \\
\hline Regular/Mal/Péssimo & $50(66,7)$ & $42(56,0)$ & $11(14,7)$ & $29(38,7)$ & $18(27,3)$ & $12(16,0)$ \\
\hline \multicolumn{7}{|l|}{ Saúde bucal [valor de $\mathrm{p}^{*}$ ] } \\
\hline Bom/ótimo & $84(73,7)$ & $82(71,9)$ & $21(18,4)$ & $42(36,8)$ & $18(18,4)$ & $19(16,7)$ \\
\hline Regular/mal/péssimo & $78(79,6)$ & $65(66,3)$ & $21(21,4)$ & $45(45,9)$ & $21(24,7)$ & $22(22,4)$ \\
\hline Consulta nutricionista [valor de $\mathrm{p}^{*}$ ] & & $\mathrm{p}<0,05$ & $\mathrm{p}<0,05$ & & $\mathrm{p}<0,05$ & $\mathrm{p}<0,05$ \\
\hline Sim & $65(74,7)$ & $67(77,0)$ & $23(26,4)$ & $33(37,9)$ & $21(28,8)$ & $23(26,4)$ \\
\hline Não & $97(77,6)$ & $80(64,0)$ & $19(15,2)$ & $54(43,2)$ & $18(16,4)$ & $18(14,4)$ \\
\hline Consulta médica no último ano [valor de $\mathrm{p}^{*}$ ] & & $\mathrm{p}<0,05$ & & & & \\
\hline $\operatorname{Sim}$ & $143(77,7)$ & $135(73,4)$ & $39(21,2)$ & $76(41,3)$ & $36(22,6)$ & $38(20,7)$ \\
\hline Não & $19(67,9)$ & $12(42,9)$ & $3(10,7)$ & $11(39,3)$ & $3(12,5)$ & $3(10,7)$ \\
\hline \multicolumn{7}{|l|}{ Número de consultas médicas [valor de $\mathrm{p}^{*}$ ] } \\
\hline Uma & $22(75,9)$ & $21(72,4)$ & $4(13,8)$ & $13(44,8)$ & $3(12,5)$ & $5(17,2)$ \\
\hline Duas ou mais & $121(78,1)$ & $114(73,5)$ & $35(22,6)$ & $63(40,6)$ & $33(24,4)$ & $33(21,3)$ \\
\hline \multicolumn{7}{|l|}{ Quem prepara as refeições [valor de $\mathrm{p}^{*}$ ] } \\
\hline Idoso & $88(77,2)$ & $85(74,6)$ & $23(20,2)$ & $44(38,6)$ & $20(20,0)$ & $22(19,3)$ \\
\hline Familiar/Cuidador/outros & $74(75,5)$ & $62(63,3)$ & $19(19,4)$ & $43(43,9)$ & $19(22,2)$ & $19(19,4)$ \\
\hline \multicolumn{7}{|l|}{ Quem compra os alimentos [valor de $\mathrm{p}^{*}$ ] } \\
\hline Idoso & $98(76,6)$ & $90(70,3)$ & $27(21,1)$ & $53(41,4)$ & $26(23,6)$ & $24(18,8)$ \\
\hline Familiar/cuidador/outros & $64(76,2)$ & $57(67,9)$ & $15(17,9)$ & $34(40,5)$ & $13(33,3)$ & $17(20,2)$ \\
\hline Motivo principal da escolha [valor de $\mathrm{p}^{*}$ ] & & $\mathrm{p}<0,05$ & $\mathrm{p}<0,05$ & & & $\mathrm{p}<0,05$ \\
\hline Hábito & $59(78,7)$ & $54(72,0)$ & $10(13,3)$ & $31(41,3)$ & $12(18,5)$ & $10(13,3)$ \\
\hline Preço & $48(71,6)$ & $39(58,2)$ & $10(14,9)$ & $27(40,3)$ & $8(14,3)$ & $9(13,4)$ \\
\hline Saudável & $37(84,1)$ & $36(81,8)$ & $16(36,4)$ & $18(40,9)$ & $14(34,1)$ & $15(34,1)$ \\
\hline Outros & $18(69,2)$ & $18(69,2)$ & $6(23,1)$ & $11(42,3)$ & $5(23,8)$ & $7(26,9)$ \\
\hline Número de refeições diárias [valor de $\mathrm{p}^{*}$ ] & & $\mathrm{p}<0,05$ & & & & \\
\hline 5 ou mais & $45(84,9)$ & $45(84,9)$ & $13(24,5)$ & $18(34,0)$ & $9(19,1)$ & $11(20,8)$ \\
\hline Menos do que 5 & $117(73,6)$ & $102(64,7)$ & $29(18,2)$ & $69(43,4)$ & $30(22,1)$ & $30(18,9)$ \\
\hline
\end{tabular}

"Qui-quadrado de Pearson; * teste de Fisher; ${ }^{* * *}$ salário mínimo: R\$ 545,00 
O que pode ser justificado pela homogeneidade da amostra quanto aos aspectos socioeconômicos e culturais. Contudo, maiores prevalências de uso de gordura vegetal e preparação de carnes assadas, cozidas ou grelhadas foram observadas entre os que já haviam consultado com nutricionista, evidenciando que as intervenções na atenção primária à saúde (APS) auxiliam na adoção de melhores hábitos alimentares pelos idosos hipertensos. Na Polônia, as mortes por DCV diminuíram $26 \%$ após uma redução substancial do consumo de gorduras animais e aumento do consumo de óleos vegetais e frutas ${ }^{25}$. Experiências semelhantes, também foram observadas em outros países ${ }^{26,27}$.

Por outro lado, o consumo regular de frutas e hortaliças teve uma elevada prevalência entre os entrevistados, semelhante ao encontrado entre idosos de Pelotas (RS) ${ }^{12}$. No entanto, essa observação é limitada, uma vez que não foram avaliadas as quantidades diárias ingeridas e nem a variedade, fatores esses diretamente associados à diminuição do risco de DCV $7,11,28$. Também observamos que o consumo diário de frutas e hortaliças foi associado a fatores socioeconômicos, o mesmo encontrado em idosos de baixa renda residentes na cidade de São Paulo ${ }^{29}$. O aumento do consumo de frutas e hortaliças é uma das metas do Plano de Ações Estratégicas para o enfrentamento das DCNT no Brasil ${ }^{10}$. Portanto, uma das maneiras de aumentar o consumo desses alimentos, é reduzindo seus preços e facilitando seu acesso. Outra medida é o incentivo ao cultivo de pomares e hortas nas residências dos idosos.

Os cereais integrais também possuem estreita ligação com a prevenção de DCV e sua ingestão deve ser estimulada, contudo, o consumo de pão integral foi pouco referido entre os idosos entrevistados ${ }^{11,30,31}$. O motivo ser saudável para escolha dos alimentos está entre os fatores que contribuíram para a maior prevalência no consumo de pão integral. No entanto, entre os que referiram como motivação para aquisição dos alimentos o hábito, foi observada menor frequência de consumo desse alimento. Portanto, o não consumo de pão integral pela maioria dos idosos hipertensos pode ser em parte justificado pela falta do hábito. Por conta disso, a promoção de oficinas que apresentem esses produtos, que trabalhem com formas de preparo e degustação, podem ser alternativas eficientes para familiarização e estimulo ao consumo.

Entre os que já haviam consultado com nutricionista, observamos maiores frequências para a maioria dos hábitos alimentares saudáveis. Contudo, ainda foi pequeno o número de idosos que já havia consultado com esse profissional. Apesar de termos encontrado um bom acesso ao atendimento médico, esse fator, isoladamente, não tem mostrado melhores desfechos clínicos e sanitários nas doenças crônicas ${ }^{32}$. Por conta disso, nossos achados reforçam a importância do trabalho multiprofissional na APS, em especial o profissional nutricionista. A carência destes profissionais na ESF é um dos fatores que dificultam a implantação da educação nutricional. Uma equipe de saúde da família, fortemente centrada na atenção do médico e do enfermeiro não dá conta de gerar todos os resultados sanitários necessários em relação às condições crônicas ${ }^{32}$.

Nossos resultados sinalizam a necessidade de investigações periódicas sobre esse tema, a fim de (re) conhecer os hábitos alimentares de grupos mais vulneráveis ao desenvolvimento de DCV e avaliar os dificultadores na adoção de hábitos alimentares saudáveis para prevenção dessas doenças. A ESF se configura como um espaço privilegiado para isto. Assim como é necessário o desenvolvimento de estudos longitudinais para melhor avaliar a associação de hábitos alimentares com DCV.

Concluindo, os resultados desse estudo sugerem que os idosos hipertensos estão encontrando dificuldade para adotar hábitos alimentares saudáveis para prevenção de DCV; contribuem ainda para a definição de estratégias de saúde pública e direcionamento dos processos de cuidado visando melhorar a qualidade de vida deles com impacto positivo nos resultados sanitários, especialmente na prevenção de doenças cardiovasculares. 


\section{Colaboradores}

SD Gadenz trabalhou na concepção do projeto, análise e interpretação dos dados, revisão crítica do conteúdo intelectual e redação do artigo. LA Benvegnú contribuiu na concepção do projeto, na análise e interpretação dos dados e na redação do artigo.

\section{Agradecimentos}

Este trabalho contou com o apoio e recurso financeiro da Fundação Municipal da Saúde de Santa Rosa. Agradecemos também a todos os idosos que participaram deste estudo e à equipe de saúde da ESF Planalto do município de Santa Rosa que ajudou a localizar os endereços.

\section{Referências}

1. Mozaffarian D, Longstreth WT Jr, Lemaitre RN, Manolio TA, Kuller LH, Burke GL, Siscovick DS. Fish consumption and stroke risk in elderly individuals: the cardiovascular health study. Arch Intern Med 2005; 165(2):200-206.

2. Schmidt MI, Duncan BB, Azevedo e Silva G, Menezes AM, Monteiro CA, Barreto SM, Chor D, Menezes PR. Chronic non-communicable diseases in Brazil: burden and current challenges. Lancet 2011; 377 (9781):1949-1961.

3. Instituto Brasileiro de Geografia e Estatística (IBGE). Censo demográfico de 2010. [site na Internet]. [acessado 2012 fev 09]. Disponível em: http://www. ibge.gov.br/home/estatistica/populacao/censo2010.

4. Vokonas PS, Kannel WB, Cupples LA. Epidemiology and risk of hypertension in the elderly: the Framingham Study. J Hypertens Suppl 1988; 6(1):S3-S9.

5. Klag MJ, Whelton PK, Appel LJ. Effect of age on the efficacy of blood pressure treatment strategies. Hypertension 1990; 16(6):700-705.

6. Geib LTC. Determinantes sociais da saúde do idoso. Cien Saude Colet 2012; 17(1):123-133.

7. American Heart Association Nutrition Committee, Lichtenstein AH, Appel LJ, Brands M, Carnethon M, Daniels S, Franch HA, Franklin B, Kris-Etherton P, Harris WS, Howard B, Karanja N, Lefevre M, Rudel L, Sacks F, Van Horn L, Winston M, WylieRosett J. Diet and lifestyle recommendations revision 2006: a scientific statement from the American Heart Association Nutrition Committee. Circulation 2006; 114(1):82-96.

8. Tourlouki E, Matalas AL, Panagiotakos DB. Dietary habits and cardiovascular disease risk in middleaged and elderly populations: a review of evidence. Clin Interv Aging 2009; 4:319-330.

9. Levy RB, Claro RM, Mondini L, Sichieri R, Monteiro CA. Regional and socioeconomic distribution of household food availability in Brazil, in 20082009. Rev Saude Publica 2012; 46(1):6-15.

10. Brasil. Ministério da Saúde (MS). Secretaria de Vigilância em Saúde. Departamento de Análise de Situação de Saúde. Plano de ações estratégicas para o enfrentamento das doenças crônicas não transmissíveis (DCNT) no Brasil 2011- 2022. Brasília: MS; 2011.

11. World Health Organization (WHO). Prevention of cardiovascular disease/: guidelines for assessment and management of cardiovascular risk. Geneva: WHO; 2007.

12. Vinholes DB, Assunção MCF, Neutzling MB. Frequência de hábitos saudáveis de alimentação medidos a partir dos 10 Passos da Alimentação Saudável do Ministério da Saúde. Pelotas, Rio Grande do Sul, Brasil. Cad Saude Publica 2009; 25(4):791-799.

13. World Health Organization (WHO). Physical status: the use and interpretation of anthropometry. Report of a WHO Expert Committee. Technical Report Series No 854. Geneva: WHO; 1995.

14. Lipschitz DA. Screening for nutritional status in the elderly. Prim Care 1994; 21(1):55-67.

15. Brasil. Ministério da Saúde (MS). Conselho Nacional de Saúde. Resolução no 196 de 10 de outubro de 1996. Diretrizes e Normas Regulamentadoras de Pesquisas Envolvendo Seres Humanos. Diário Oficial da União 1996; 16 out. 
16. Zaitune MP, Barros MB, César CL, Carandina L, Goldbaum M. Hipertensão arterial em idosos: prevalência, fatores associados e práticas de controle no Município de Campinas, São Paulo, Brasil. Cad Saude Publica 2006; 22(2):285-294.

17. Nunes DP, Nakatani AYK, Silveira EA, Bachion MM, de Souza MR. Capacidade funcional, condições socioeconômicas e de saúde de idosos atendidos por equipes de Saúde da Família de Goiânia (GO, Brasil). Cien Saude Colet 2010; 15(6):2887-2898.

18. Instituto Brasileiro de Geografia e Estatística (IBGE). Departamento de População e Indicadores Sociais. Perfil dos idosos responsáveis pelos domicílios no Brasil, 2000. Rio de Janeiro: IBGE; 2002.

19. Minardi-Cotta RM, Batista KCS, Reis RS, de Souza GA, Dias G, de Castro FAF Alfenas R de C. Perfil sociossanitário e estilo de vida de hipertensos e/ou diabéticos, usuários do Programa de Saúde da Família no município de Teixeiras, MG. Cien Saude Colet 2009; 14(4):1251-1260.

20. Sierra-Johnson J, Undén A-L, Linestrand M, Rosell M, Sjogren P, Kolak M, De Faire U, Fisher RM, Hellénius ML. Eating meals irregularly: a novel environmental risk factor for the metabolic syndrome. Obesity (Silver Spring) 2008; 16(6):1302-1307.

21. Redondo MR, Ortega RM, Zamora MJ, Quintas ME, López-Sobaler AM, Andrés P, Gaspar MJ. Influence of the number of meals taken per day on cardiovascular risk factors and the energy and nutrient intakes of a group of elderly people. Int $J$ Vitam Nutr Res 1997; 67(3):176-182.

22. Titan SM, Bingham S, Welch A, Luben R, Oakes S, Day N, Khaw KT. Frequency of eating and concentrations of serum cholesterol in the Norfolk population of the European prospective investigation into cancer (EPIC-Norfolk): cross sectional study. BMJ 2001; 323(7324):1286-1288.

23. Brasil. Ministério da Saúde (MS). Secretaria de Atenção à Saúde. Departamento de Atenção Básica. Alimentação saudável para a pessoa idosa: um manual para profissionais de saúde. Brasília: MS; 2009. (Série A. Normas e Manuais Técnicos).

24. Morrison AC, Ness RB. Sodium intake and cardiovascular disease. Annu Rev Public Health 2011; 32:7190.

25. Zatonski WA, Willett W. Changes in dietary fat and declining coronary heart disease in Poland: population based study. BMJ 2005; 331(7510):187-188.

26. Dowse GK, Gareeboo H, Alberti KG, Zimmet P, Tuomilehto J, Purran A, Fareed D, Chitson P, Collins VR. Changes in population cholesterol concentrations and other cardiovascular risk factor levels after five years of the non-communicable disease intervention programme in Mauritius. Mauritius Noncommunicable Disease Study Group. BMJ 1995; 311(7015):1255-1259.

27. Laatikainen T, Critchley J, Vartiainen E, Salomaa V, Ketonen M, Capewell S. Explaining the decline in coronary heart disease mortality in Finland between 1982 and 1997. Am J Epidemiol 2005; 162(8):764-773.
28. Mancia G, De Backer G, Dominiczak A, Cifkova R, Fagard R, Germano G, Grassi G, Heagerty AM, Kjeldsen SE, Laurent S, Narkiewicz K, Ruilope L, Rynkiewicz A, Schmieder RE, Boudier HA, Zanchetti A, Vahanian A, Camm J, De Caterina R, Dean V, Dickstein K, Filippatos G, Funck-Brentano C, Hellemans I, Kristensen SD, McGregor K, Sechtem U, Silber S, Tendera M, Widimsky P, Zamorano JL, Erdine S, Kiowski W, Agabiti-Rosei E, Ambrosioni E, Lindholm LH, Viigimaa M, Adamopoulos S, Agabiti-Rosei E, Ambrosioni E, Bertomeu V, Clement D, Erdine S, Farsang C, Gaita D, Lip G, Mallion JM, Manolis AJ, Nilsson PM, O’Brien E, Ponikowski P, Redon J, Ruschitzka F, Tamargo J, van Zwieten P, Waeber B, Williams B. Management of Arterial Hypertension of the European Society of Hypertension; European Society of Cardiology. 2007 Guidelines for the Management of Arterial Hypertension: The Task Force for the Management of Arterial Hypertension of the European Society of Hypertension (ESH) and of the European Society of Cardiology (ESC). J Hypertens 2007; 25(6):1105-1187.

29. Viebig RF, Pastor-Valero M, Scazufca M, Menezes PR. Fruit and vegetable intake among low income elderly in the city of São Paulo, Southeastern Brazil. Rev Saude Publica 2009; 43(5):806-813.

30. Mozaffarian D, Kumanyika SK, Lemaitre RN, Olson JL, Burke GL, Siscovick DS. Cereal, fruit, and vegetable fiber intake and the risk of cardiovascular disease in elderly individuals. JAMA 2003; 289(13): 1659-1666.

31. De Backer G, Ambrosioni E, Borch-Johnsen K, Brotons C, Cifkova R, Dallongeville J Ebrahim S, Faergeman O, Graham I, Mancia G, Manger Cats V, Orth-Gomér K, Perk J, Pyörälä K, Rodicio JL, Sans S, Sansoy V, Sechtem U, Silber S, Thomsen T, Wood D, Third Joint Task Force of European and Other Societies on Cardiovascular Disease Prevention in Clinical Practice. European guidelines on cardiovascular disease prevention in clinical practice. Third Joint Task Force of European and Other Societies on Cardiovascular Disease Prevention in Clinical Practice. Eur Heart J 2003; 24(17):1601-1610.

32. Mendes EV. O cuidado das condições crônicas na atenção primária à saúde: o imperativo da consolidação da estratégia da saúde da família. Brasília: Organização Pan-Americana da Saúde (OPAS); 2012.

Artigo apresentado em 18/08/2012

Aprovado em 30/09/2012

Versão final apresentada em 05/10/2012 
